\title{
A Hubble constant measurement from superluminal mo- tion of the jet in GW170817
}

K. Hotokezaka (Princeton), E. Nakar (Tel Aviv), O. Gottlieb (Tel Aviv), S. Nissanke (GRAPPA University of Amsterdam, Nikhef, Radboud), K. Masuda (NASA Sagan Fellow, Princeton), G. Hallinan (Caltech), K. P. Mooley (Jansky Fellow, NRAO/Caltech), A. T. Deller (Swinburne, OzGrav)

The Hubble constant $\left(H_{0}\right)$ measures the current expansion rate of the Universe, and plays a fundamental role in cosmology. Tremendous effort has been dedicated over the past decades to measure $H_{0}^{11-10}$. Notably, Planck cosmic microwave background (CMB) and the local Cepheid-supernovae distance ladder measurements determine $H_{0}$ with a precision of $\sim 1 \%$ and $\sim 2 \%$ respectively $\sqrt{3 / 4 / 11}$. A 3- $\sigma$ level of discrepancy exists between the two measurements ${ }^{4 / 12}$, for reasons that have yet to be understood. Gravitational wave (GW) sources accompanied by electromagnetic (EM) counterparts offer a completely independent standard siren (the GW analogue of an astronomical standard candle) measurement of $H_{0} 13-15$, as demonstrated following the discovery of the neutron star merger, GW17081 $7^{16-18}$. This measurement does not assume a cosmological model and is independent of a cosmic distance ladder. The first joint analysis of the GW signal from GW170817 and its EM localization led to a measurement of $H_{0}=74_{-8}^{+16} \mathbf{~ k m / s / M p c ~ ( m e d i a n ~ a n d ~ s y m m e t r i c ~} 68 \%$ credible interval) ${ }^{15}$. In this analysis, the degeneracy in the GW signal between the source distance and the weakly constrained viewing angle dominated the $H_{0}$ measurement uncertainty. Recently, Mooley et al. (2018) 
obtained tight constraints on the viewing angle using high angular resolution imaging of the radio counterpart of GW170817. Here we obtain a significantly improved measurement $H_{0}=68.9_{-4.6}^{+4.7} \mathbf{~ k m / s / M p c ~ b y ~ u s i n g ~ t h e s e ~ n e w ~ r a d i o ~ o b s e r v a t i o n s , ~ c o m b i n e d ~ w i t h ~ t h e ~ p r e v i o u s ~}$ GW and EM data. We estimate that 15 more localized GW170817-like events (comparable signal-to-noise ratio, favorable orientation), having radio images and light curve data, will potentially bring resolution to the tension between the Planck and Cepheid-supernova measurements, as compared to 50-100 GW events without such data ${ }^{2021}$.

Mooley et al. $(2018)^{19}$ recently obtained the radio images of a narrowly collimated jet associated with GW170817 by using Very Long Baseline Interferometer (VLBI) and reported the centroid motion of $2.7 \pm 0.3$ mas from day 75 to 230 , indicating the superluminal motion of the jet at an apparent velocity $\beta_{\mathrm{app}}=(4.1 \pm 0.4)\left(\frac{d}{41 \mathrm{Mpc}}\right)$, where $d$ is the source distance from Earth and the velocity is in units of the speed of light, $c$. In addition, the slow rise $\frac{22-24}{24}$ and fast decline $\frac{19,25 \mid 26}{2}$ of the afterglow light curve provide us with evidence that a narrowly collimated jet dominates the emission after the light curve peak. These observations allow us to determine the observing angle independently of the GW analysis.

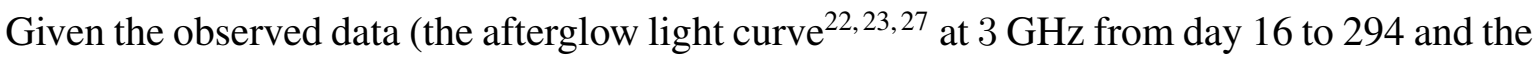
centroid motion ${ }^{19}$ ), we constrain the observing angle using several methods: analytic modelling, full hydrodynamic numerical simulations and semi-analytic calculations of synthetic jet models. The analytic modelling and numerical simulations are described in Mooley et al. $(2018)^{19}$. Giventhe importance of the new constraints on the observing angle on our results, we give here a brief 
summary of their results. Mooley et al. (2018) find that the model which best fits the observations is that of a successful jet. We define $\theta_{j}$ as the jet opening angle, $\theta_{\text {obs }}$ as the observing angle, and the difference between them as $\delta_{\theta}=\theta_{\text {obs }}-\theta_{j}$. Mooley et al. (2018) ${ }^{19}$ show that the light curve and the small image size imply that the jet must be very narrow, i.e., $\theta_{j} \ll \delta_{\theta}$. This implies that the superluminal motion of the jet image can be approximated as that of a point source, where $\delta_{\theta} \approx 1 / \Gamma$ at the time of the observations (near the peak of the light curve). This implies $\delta_{\theta} \approx 1 / \beta_{\mathrm{app}} \approx 0.25 \mathrm{rad}$ and $\theta_{j} \ll 0.25 \mathrm{rad}$, where a source distance of $41 \mathrm{Mpc}$ is assumed. In order to verify this conclusion and to quantify the allowed region for $\delta_{\theta}$ and $\theta_{j} \ll 0.25 \mathrm{rad}$, they then carried out a set of numerical simulations varying both the opening angle of the jet and the viewing angle allowing for a systematic check of which models can fit both the light curve and the images. They find that only models with $1 / 5<\delta_{\theta}<1 / 3 \mathrm{rad}$ and $\theta_{j}<0.1 \mathrm{rad}$ are consistent with observations. They conclude that the combination of the VLBI measurements and the light curve dictates $0.25<\theta_{\text {obs }}<0.45 \mathrm{rad}\left(15^{\circ}<\theta_{\text {obs }}<25^{\circ}\right)$. This constraint is derived assuming that the distance to the source, $d$, is known $(41 \mathrm{Mpc})$. However, in our analysis the distance is unknown and since the main constraint on the observing angle is derived from the the apparent velocity, $\beta_{\text {app }} \propto d$, the observing angle is constrained to $0.25<\theta_{\text {obs }}\left(\frac{d}{41 \mathrm{Mpc}}\right)<0.45 \mathrm{rad}$.

In order to obtain the probability distribution of $\theta_{\text {obs }}$ and $d$, and to estimate the effect of the jet modelling on the observational constraints on the opening angle, we run also Markov chain Monte Carlo simulations with two synthetic jet models: a Power-Law Jet (PLJ) and a Gaussian Jet (GJ; see Method). While the hydrodynamics of the jet is not fully taken into account in the synthetic models, unlike the numerical simulations, they allow us to scan the entire parameter space. 
Therefore, this analysis and the estimate based on the hydrodynamic simulations ${ }^{19}$ are complementary. Figure 1 shows the posterior distribution for $d$ and $\theta_{\text {obs }}$ (see Methods). The observing angle is constrained to $0.29_{-0.01}^{+0.02} \mathrm{rad}$ and $0.30_{-0.02}^{+0.02} \mathrm{rad}$ for PLJ and GJ models, respectively. The constraint on the observing angle for a given model is tighter than the one obtained by the hydrodynamical simulations, most likely because the simulations explore various outflow structures while each synthetic model explores a single outflow shape. The most likely observing angles found with the synthetic models are smaller by $\sim 0.05$ rad than the median based on the hydrodynamic simulations (but still within the errors). We consider this difference as a systematic uncertainty of our analysis (elaborated below), which is most likely attributed to the partial treatment of the hydrodynamic evolution.

We now turn to the combined GW-EM analysis of the Hubble constant $\left(H_{0}\right)$. Namely, we combine the 2-dimensional marginalized GW likelihood distribution (high spin PhenomPNRT) ${ }^{28}$ for $d$ and $\theta_{\text {obs }}$ with that determined from the afterglow light curve and centroid motion (see Methods). The posterior distribution for $H_{0}$ is then computed from the combined likelihood for $d$ and the information about the host galaxy NGC4993 (see Methods) $\sqrt{15}$. Figure 2 depicts the posterior distribution for $H_{0}$ for a PLJ model and that of the GW-only analysis $\frac{15 / 28}{5}$. The constraint is improved from the GW-only analysis, $74_{-8}^{+16} \mathrm{~km} / \mathrm{s} / \mathrm{Mpc}$, to $68.3_{-4.3}^{+4.4} \mathrm{~km} / \mathrm{s} / \mathrm{Mpc}$ (median and symmetric $68 \%$ credible interval). Also depicted in Figure 2 are the regions determined by the Planck $\mathrm{CMB}^{3}$ and SHOES Cepheid-supernova distance ladder ${ }^{4}$ surveys respectively. Figure 3 shows the posterior distributions for $H_{0}$ with the different jet models: hydrodynamics simulation jet $\left(0.25<\theta_{\mathrm{obs}}\left(\frac{d}{41 \mathrm{Mpc}}\right)<0.45 \mathrm{rad}\right)$, PLJ, and GJ models. The medians and $68 \%$ credible 
intervals are $68.9_{-4.5}^{+4.6}, 68.3_{-4.3}^{+4.4}$, and $68.5_{-4.3}^{+4.4} \mathrm{~km} / \mathrm{s} / \mathrm{Mpc}$, respectively, corresponding to a precision of $6-7 \%$ at $1-\sigma$ level. The sources of errors in our analysis are the GW data, the shape of the light curve, the centroid motion, and the peculiar velocity of the host galaxy. While the constraint on $\theta_{\text {obs }}$ is slightly different between the three models, the systematic error in $H_{0}$ due to this difference is much smaller than $7 \%$. This is because the uncertainty in $H_{0}$ of our analysis is dominated by both the GW data and the peculiar motion of NGC 4993 (contrary to the GW-only analysis, where the uncertainty in the observing angle is a major source of error). Finally, it is important to bear in mind that our result does not depend on the spin prior in the GW analysis ${ }^{28}$ (see Methods).

Our new analysis, which is based on this single event, improves the $H_{0}$ measurement to a precision of $\sim 7 \%$. We expect that the precision of the measurement will improve by observing more merger events similar to GW170817, i.e, mergers with detectable jet afterglows. In the coming years, several to tens of neutron star binary mergers (including neutron star-black hole binary systems) per year may be observable in GWs as the LIGO and Virgo detectors improve their sensitivity due to instrument upgrades, and as additional detectors join the GW network ${ }^{29}$. In addition, radio afterglow fluxes of merger events at further distances are not necessarily fainter than GW170817 because of the wide variation in the circum-merger densities. For instance, the superluminal motion of a jet can be measured for events taking place out to $\sim 100 \mathrm{Mpc}$ if the density is about the typical value inferred from short GRB observations ${ }^{30}$ (and the other afterglow parameters are assumed to be the same as GW170817). We note however that a favorable viewing angle is a likely prerequisite for detection. For events at greater $d$, while the error due to the radio observations increases, the error due to the peculiar motion decreases. Furthermore, inferring 
the binary inclination from GW-alone relies on the measurement of the GW polarization, which was particularly challenging in the case of GW170817 because of the low signal-to-noise ratio in the Virgo detector and the two LIGO detectors being nearly co-aligned ${ }^{15 / 16}$. For future GW radio jet events with similar signal-to-noise, the $H_{0}$ uncertainty would thus remain comparable or better to that of this analysis because of the addition of GW detectors and of improved instrument sensitivity 2021/31. To achieve a measurement of $H_{0}$ with a high precision using more events, the systematic uncertainty resulting from jet modeling should also be reduced.

Most current methods to estimate $H_{0}$ span from the local Universe to the CMB and include the use of Cepheid variables and red-giant stars $^{7}$, supernovae $(\mathrm{SNe})^{4 / 5 \mid 11}$, circumnuclear megamasers ${ }^{6}$, gravitational lenses ${ }^{8}$, galaxies ${ }^{2910}$ and the $\mathrm{CMB}^{13}$. These methods either depend on a cosmic distance ladder relating geometric distances of Cepheid variables to standard candles, such as Type 1a supernovae, or assume a certain cosmological model, such as $\Lambda$-CDM $1+5,7+10$. The use of geometric distances to circumnuclear megamasers is a notable exception, but is currently limited to $6 \%$ precision $^{6}$. The current $\gtrsim 3 \sigma$ discrepancy ${ }^{4.12}$ between Planck CMB measurements and SHOES data is of particular interest given the degree of precision in both measurements and the possible implication of the requirement of new physics beyond $\Lambda$-CDM models if the discrepancy turns out to be true (rather then a result of systematic errors) $\sqrt{32}$. Gaia DR2 data on Galactic Cepheids, together with dedicated $H S T$ observations on the latter sample, will likely reduce systematic uncertainties sufficiently to improve the standard candle/distance ladder measurements of $H_{0}$ to $\sim 1 \%$ precision within the next few years $\frac{11}{11}$, potentially raising this discrepancy above $5 \sigma$. A standard siren based measurement of $H_{0}$, on a similar timescale, would be particularly useful, as 
it would independently provide a local measurement of $H_{0}$ that does not rely on a cosmic distance ladder, and which does not assume any cosmological model as a prior (although there are model assumptions in the interpretation of the VLBI data). We estimate that, after observing $\sim 15$ more GW 170817-like events with VLBI data and light curve (comparable SNR, favorable orientation), as compared to $\sim 50-100 \mathrm{GW}$ events without such data, the precision of the $H_{0}$ measurement would be $\sim 1.8 \% 20[21] 31$. Thus, joint GW-VLBI constraints on $H_{0}$ will potentially resolve the current tension between Planck and standard candle/distance ladder data. 


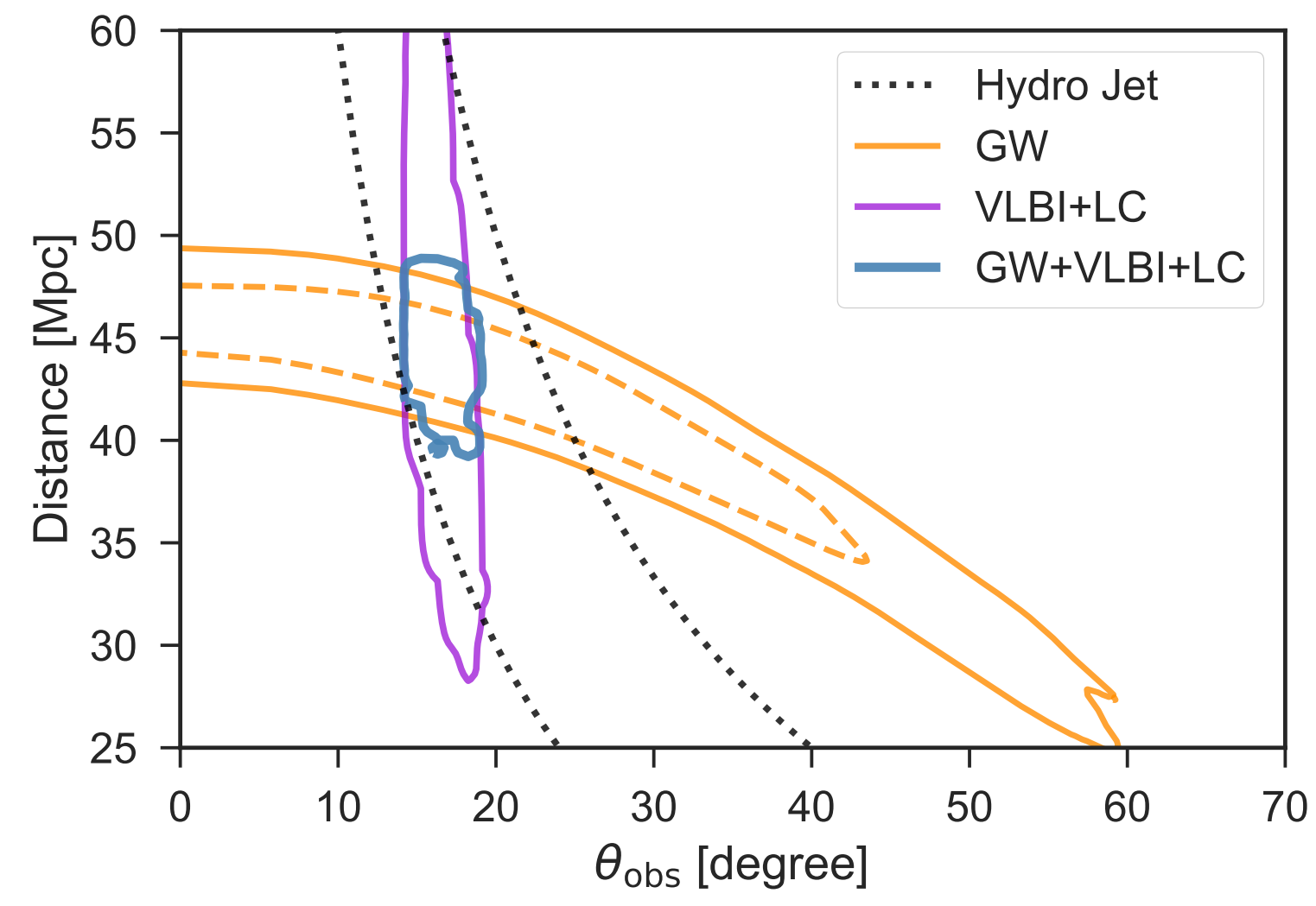

Figure 1: Distance and observing angle constraints to GW170817. Dashed curves running from top to bottom depict the constraint of $0.25<\theta_{\text {obs }}\left(\frac{d}{41 \mathrm{Mpc}}\right)<0.45 \mathrm{rad}$ estimated based on

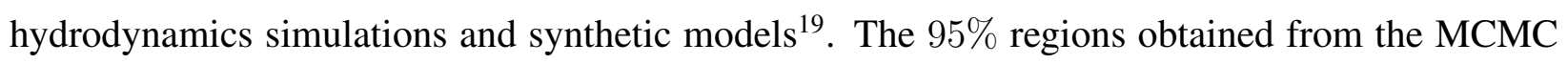
analysis of the afterglow light curve (LC) and centroid motion through Very Long Baseline Interferometry (VLBI) are shown as solid purple (VLBI+LC). The blue contours (VLBI+LC+GW) is the same, but also combined with the GW analysis for a PLJ model. Also shown as an orange dashed (solid) contour is the $68(95 \%)$ contour of the posterior distribution of the GW-only analysis (high spin PhenomPNRT posterior samples) $\stackrel{28}{28}$. We note that the VLBI and light curve data alone provide a distance estimate independent of all other means. 


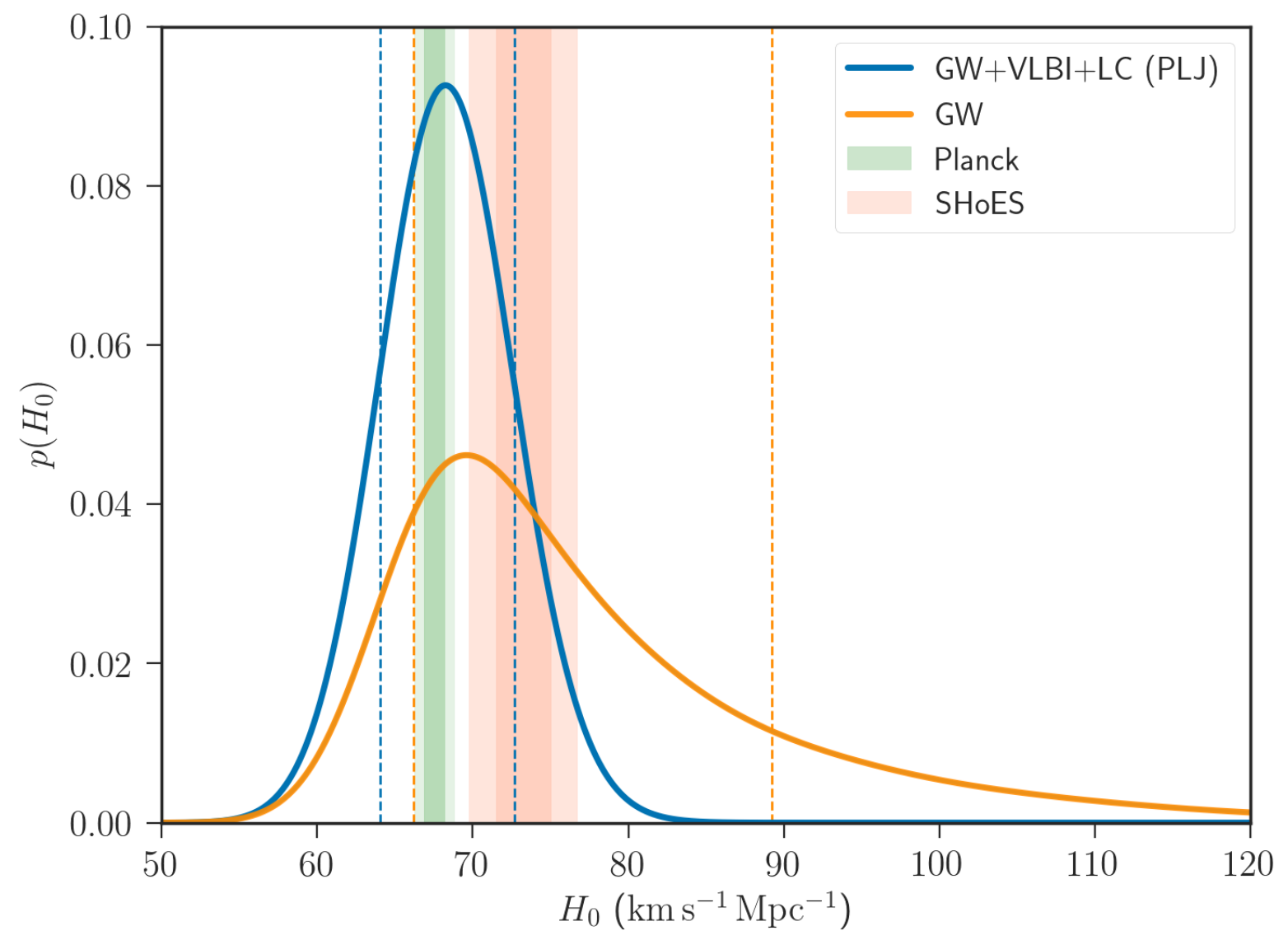

Figure 2: Posterior distributions for $H_{0}$. The results of the GW-only analysis and the combined GW-EM analysis with a PLJ model are shown. The vertical dashed lines show symmetric $68 \%$ credible interval for each model. The 1 and $2-\sigma$ regions determined by Planck CMB (TT,TE,EE+lowP+lensing) ${ }^{\sqrt{3}}$ (green) and SHOES Cepheid-SN distance ladder surveys ${ }^{4}$ (orange) are also depicted as vertical bands. 


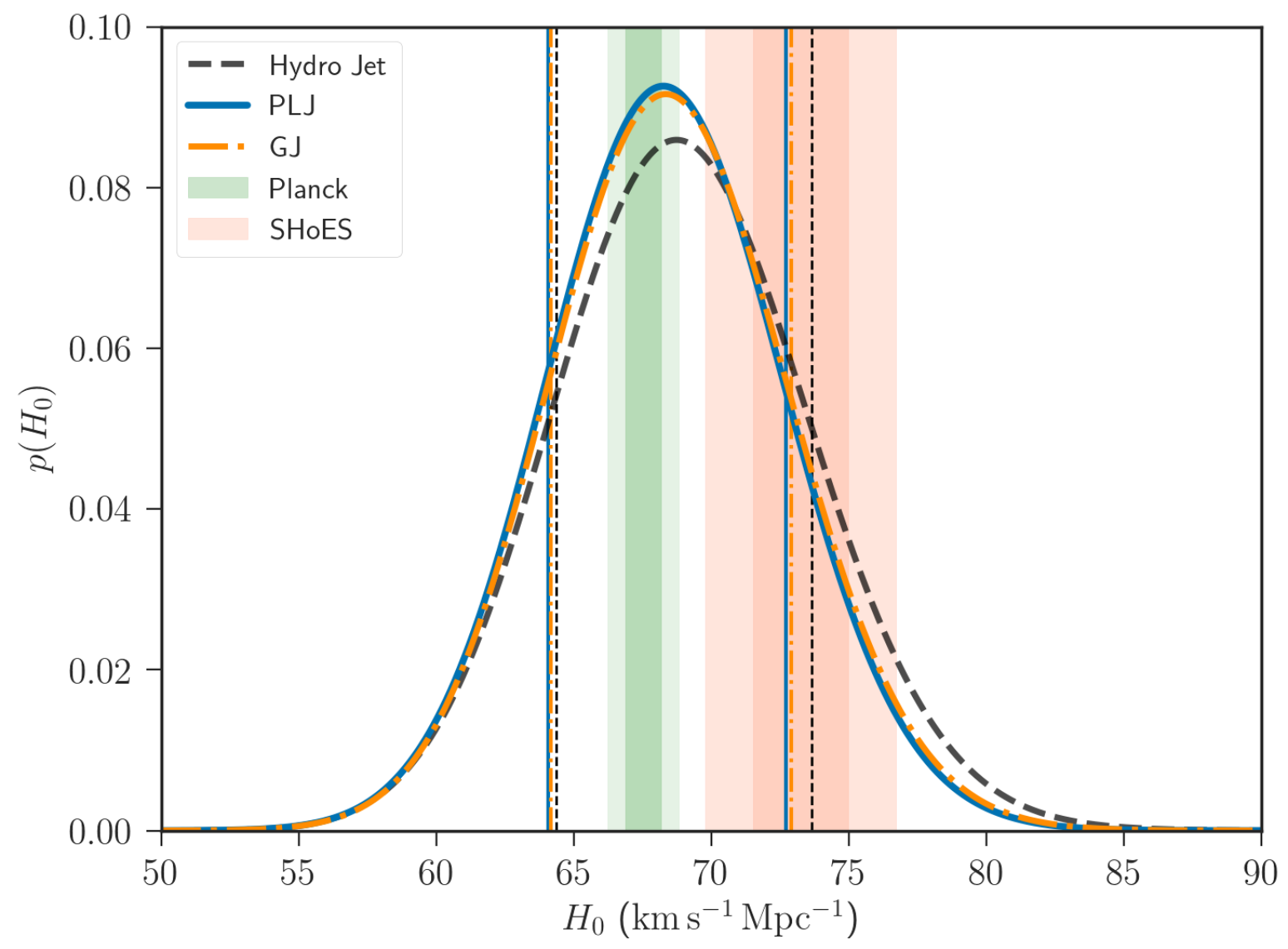

Figure 3: The Hubble constant with different jet models. Dashed curve: hydrodynamics simulation jet $\left(0.25<\theta_{\mathrm{obs}}\left(\frac{d}{41 \mathrm{Mpc}}\right)<0.45 \mathrm{rad}\right)$, solid curve: a Power-Law Jet, and dash-dotted curve: a Gaussian Jet. The vertical lines show symmetric $68 \%$ credible interval for each model. The 1 and 2- $\sigma$ regions determined by Planck CMB (TT,TE,EE+lowP+lensing) ${ }^{\sqrt{3}}$ (green) and SHOES Cepheid-SN distance ladder surveys ${ }^{4}$ (orange) are also depicted as vertical bands. 


\section{Methods}

Light curve and centroid motion modeling In the case of the afterglow of GW170817, the observed light curve rules out the simple top-hat jet model and support structured jet models $\sqrt{22+\sqrt[24]{26}} \cdot 33 \sqrt[40]{4}$, of which the structure is likely composed of the jet core and surrounding cocoon $\sqrt{19,40}-42$. We use two different structured jet models: (1) a Power-Law Jet (PLJ) and (2) a Gaussian Jet (GJ) model, which can mimic the jet-cocoon structure obtained from numerical simulations $19,40,42$. The isotropic-equivalent energy and initial Lorentz factor vary with the polar angle for a PLJ model:

$$
\begin{aligned}
E_{\mathrm{iso}}(\theta) & =\frac{E_{\mathrm{iso}, c}}{1+\left(\theta / \theta_{c}\right)^{\alpha_{E}}}, \\
\Gamma_{i}(\theta) & =1+\frac{\Gamma_{i, c}}{1+\left(\theta / \theta_{c}\right)^{\alpha_{g}}},
\end{aligned}
$$

where $E_{\mathrm{iso}, c}, \theta_{c}, \alpha_{E}$, and $\alpha_{g}$ are free parameters and we fix $\Gamma_{i, c}$ to be 600 . For a GJ model:

$$
\begin{aligned}
E_{\mathrm{iso}}(\theta) & =E_{\mathrm{iso}, c} \exp \left[-\frac{1}{2}\left(\frac{\theta}{\theta_{c}}\right)^{2}\right] \\
\Gamma_{i}(\theta) & =1+\left(\Gamma_{i, c}-1\right) \exp \left[-\frac{1}{2}\left(\frac{\theta}{\theta_{c}}\right)^{2}\right],
\end{aligned}
$$

where $E_{\mathrm{iso}, c}, \theta_{c}$ are free parameters and we fix $\Gamma_{i, c}$ to be 100 .

For a given set of the model parameters and circum-merger density, $n$, we evolve the jet adiabatically and neglect the lateral expansion ${ }^{37}$. This assumption is valid until the jet slows down sufficiently. For the core of the jet, the lateral expansion occurs on a time scale much longer than what we have considered here, and indeed, we find lack of significant lateral expansion also the hydrodynamical simulations ${ }^{19}$. For the wing of the jet, however, the lateral expansion is important on the time scales considered here ${ }^{19}$. Therefore, our approximation here is expected to slightly 
underestimate the observing angle.

Given a jet evolution, we calculate the afterglow light curve and the motion of the flux center by using the standard synchrotron afterglow model ${ }^{43}$. The code is described in Hotokezaka and Piran $(2015)^{44}$. In the case of GW170817, the afterglow has a single power-law spectrum with a spectral index of $0.588 \pm 0.005$ from radio to X-ray band $[25[26] 34$, which is consistent with optically thin synchrotron emission in the slow cooling regime. Thus, here we consider only this regime. The synchrotron modeling involves three microphysics parameters $\left(p, \epsilon_{e}, \epsilon_{b}\right)$, where $\epsilon_{e}$ and $\epsilon_{b}$ are the conversion efficiency from the internal energy to the energy of accelerated electrons and magnetic field, and $p$ is the power-law index of the number distribution of accelerated electrons. Since the power-law index, $\mathrm{p}$, is related to the observed spectrum as $F_{\nu} \propto \nu^{-(p-1) / 2}$, we adopt $p=2.16$. We also fix $\epsilon_{e}$ to be 0.1 .

Assuming the above models, we run Markov-Chain Monte Carlo (MCMC) simulations by using an open code emcee $e^{45}$. For the modelling, we use $E_{\text {iso }, c} / n$, which determines the deceleration time scale of the jet, instead of using $E_{\mathrm{iso}, c}$ and $n$ separately to reduce the number of free parameters. Furthermore, instead of using $\epsilon_{b}$, we introduce an auxiliary parameter, $e_{b}$, which controls the overall amplitude of the light curve. Therefore, in total, we have 7 parameters $\left(E_{\text {iso }, c} / n\right.$, $\left.\theta_{c}, \alpha_{E}, \alpha_{g}, e_{b}, \theta_{\mathrm{obs}}, d\right)$ for PLJ model and 5 parameters $\left(E_{\mathrm{iso}, c} / n, \theta_{c}, e_{b}, \theta_{\mathrm{obs}}, d\right)$ for GJ model. We adopt a $\log$ flat prior for $E_{\mathrm{iso}, c} / n$ and $e_{b}$, and uniform prior for $\theta_{c}, \alpha_{E}, \alpha_{g}$, an isotropic prior for $\theta_{\text {obs }}$, and a volumetric prior for $d$.

Figure 1 $\left(\right.$ VLBI+LC) shows the resulting posterior for $d$ and $\theta_{\text {obs }}$ marginalized over the other 
model parameters. The corner plots for the model parameters are shown in Extended Data Figures 1 and 2 .

Combined GW-EM analysis of the Hubble constant Next we perform the modeling of the light curve $x_{\mathrm{LC}}$ and centroid motion data $x_{\mathrm{VLBI}}$, taking into account the constraint from the $\mathrm{GW}$ data $x_{\mathrm{GW}}$. Because the GW and EM data are independent and only $d$ and $\theta_{\mathrm{obs}}$ in the GW model affect the EM data, this can be done by replacing the prior on $d$ and $\theta_{\text {obs }}$ in the above MCMC analysis with the marginal posterior distribution from the $\mathrm{GW}$ analysis, $p\left(d, \theta_{\mathrm{obs}} \mid x_{\mathrm{GW}}\right)$. Figure $1(\mathrm{GW}+\mathrm{VLBI}+\mathrm{LC})$ shows the resulting posterior distribution $p\left(d, \theta_{\mathrm{obs}} \mid x_{\mathrm{GW}}, x_{\mathrm{VLBI}}, x_{\mathrm{LC}}\right)$ marginalized over the other model parameters. The corresponding corner plots for the model parameters are shown in Extended Data Figures 4 and 5. The posterior models for the afterglow flux at $3 \mathrm{GHz}$ and centroid motion from day 75 to 230 measured with VLBI $1922 \sqrt[23]{23}$ are shown in Extended Data Figure 3 with the data.

We combine $p\left(d, \theta_{\mathrm{obs}} \mid x_{\mathrm{GW}}, x_{\mathrm{VLBI}}, x_{\mathrm{LC}}\right)$ from this joint modeling with the recessional velocity $v_{\mathrm{r}}$ to derive the Hubble constant $H_{0}$. To do so, one needs to take into account the unknown peculiar velocity of NGC 4993 as $v_{\mathrm{r}}=H_{0} d+v_{\mathrm{p}}$. Here we follow the procedure used in Abbott et al. (2017) ${ }^{\frac{15}{6}}$ to compute the marginalized posterior for $H_{0}$ :

$$
\begin{aligned}
& p\left(H_{0} \mid x_{\mathrm{GW}}, x_{\mathrm{VLBI}}, x_{\mathrm{LC}}, v_{\mathrm{r}},\left\langle v_{\mathrm{p}}\right\rangle\right) \\
& =\int \mathrm{d} d \mathrm{~d} \cos \theta_{\mathrm{obs}} \mathrm{d} v_{\mathrm{p}} p\left(H_{0}, d, \cos \theta_{\mathrm{obs}}, v_{\mathrm{p}} \mid x_{\mathrm{GW}}, x_{\mathrm{VLBI}}, x_{\mathrm{LC}}, v_{\mathrm{r}},\left\langle v_{\mathrm{p}}\right\rangle\right) \\
& \propto p\left(H_{0}\right) \int \mathrm{d} d \mathrm{~d} v_{\mathrm{p}} p\left(v_{\mathrm{r}} \mid d, v_{\mathrm{p}}, H_{0}\right) p\left(\left\langle v_{\mathrm{p}}\right\rangle \mid v_{\mathrm{p}}\right) p\left(v_{\mathrm{p}}\right) p\left(d \mid x_{\mathrm{GW}}, x_{\mathrm{VLBI}}, x_{\mathrm{LC}}\right) .
\end{aligned}
$$


We adopt the same information on $v_{\mathrm{r}}$ and $\left\langle v_{\mathrm{p}}\right\rangle$ as in (Abbott et al 2017) $)^{15}$ :

$$
\begin{aligned}
p\left(v_{\mathrm{r}} \mid d, v_{\mathrm{p}}, H_{0}\right) & =\frac{1}{\sqrt{2 \pi \sigma_{v_{r}}^{2}}} \exp \left[-\frac{1}{2}\left(\frac{v_{\mathrm{r}}-v_{\mathrm{p}}-H_{0} d}{\sigma_{v_{r}}}\right)^{2}\right], \\
p\left(\left\langle v_{\mathrm{p}}\right\rangle \mid v_{\mathrm{p}}\right) & =\frac{1}{\sqrt{2 \pi \sigma_{v_{p}}^{2}}} \exp \left[-\frac{1}{2}\left(\frac{\left\langle v_{\mathrm{p}}\right\rangle-v_{\mathrm{p}}}{\sigma_{v_{p}}}\right)^{2}\right],
\end{aligned}
$$

where $v_{\mathrm{r}}=3327 \mathrm{~km} / \mathrm{s}, \sigma_{v_{r}}=72 \mathrm{~km} / \mathrm{s},\left\langle v_{\mathrm{p}}\right\rangle=310 \mathrm{~km} / \mathrm{s}$, and $\sigma_{v_{p}}=150 \mathrm{~km} / \mathrm{s}$.

The posterior distribution for $H_{0}$ generally depends on the prior in the GW analysis ${ }^{28}$, i.e., the high or low spin prior. Figure 6 compares the $H_{0}$ posterior of the high spin prior with that of the low spin prior ${ }^{28}$. In the case of the GW-only analysis, they depend on the prior as $78_{-10}^{+20}$ $\mathrm{km} / \mathrm{s} / \mathrm{Mpc}$ (low spin) and $74_{-8}^{+15} \mathrm{~km} / \mathrm{s} / \mathrm{Mpc}$ (high spin). However, in the case of the combined analysis, they result in practically the same $H_{0}, 68.9_{-4.5}^{+4.6} \mathrm{~km} / \mathrm{s} / \mathrm{Mpc}$. We also did the same analysis by using the GW posterior data of Finstad et al. (2018) of $H_{0}$ compared to those with Abbott et al $(2018)^{28}$. Note also that our result is consistent with $H_{0}=71.9 \pm 7.1 \mathrm{~km} / \mathrm{s} / \mathrm{Mpc}$ measured by using the surface brightness fluctuation method applied to NGC 499377, which is calibrated with the Cepheid distance measurements. 
Extended Data 


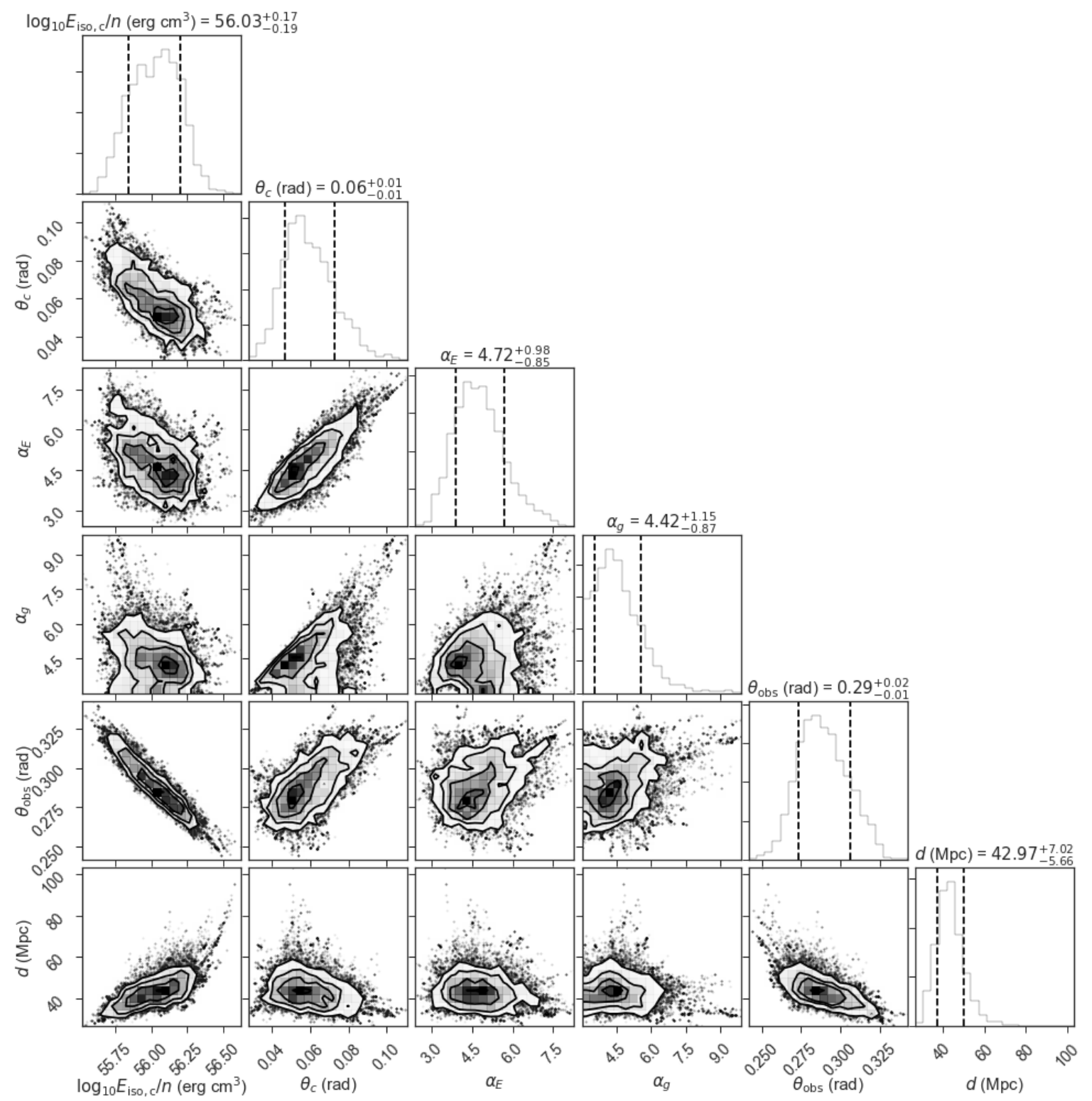

Figure 1: Corner plot ${ }^{48}$ for a Power-Law Jet model. The afterglow light curve at $3 \mathrm{GHz}$ and the centroid motion resolved by VLBI are used as the observed input data. Vertical lines depict $68 \%$ credible intervals. 


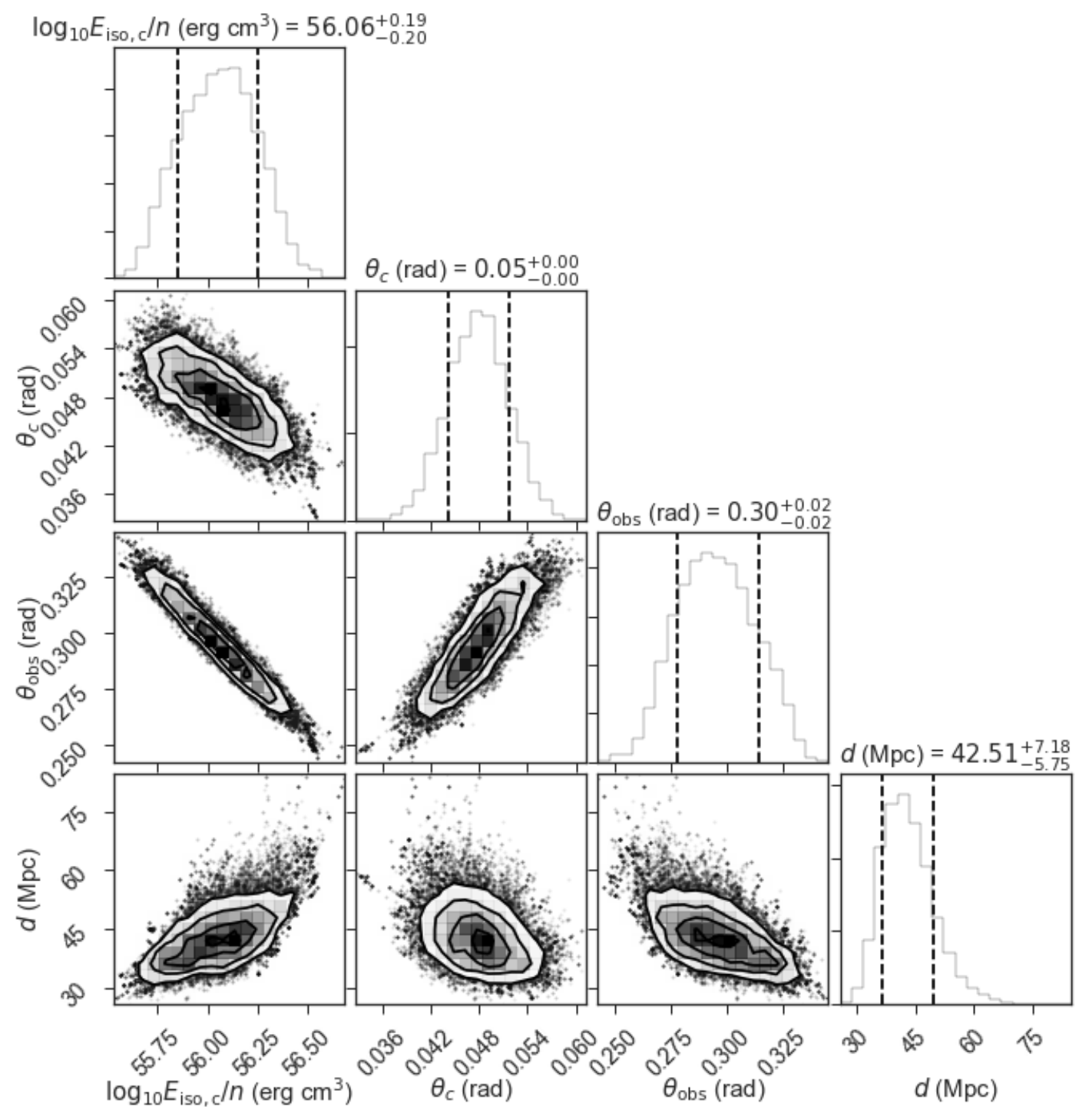

Figure 2: Same as Figure 1 but for a Gaussian Jet model. 

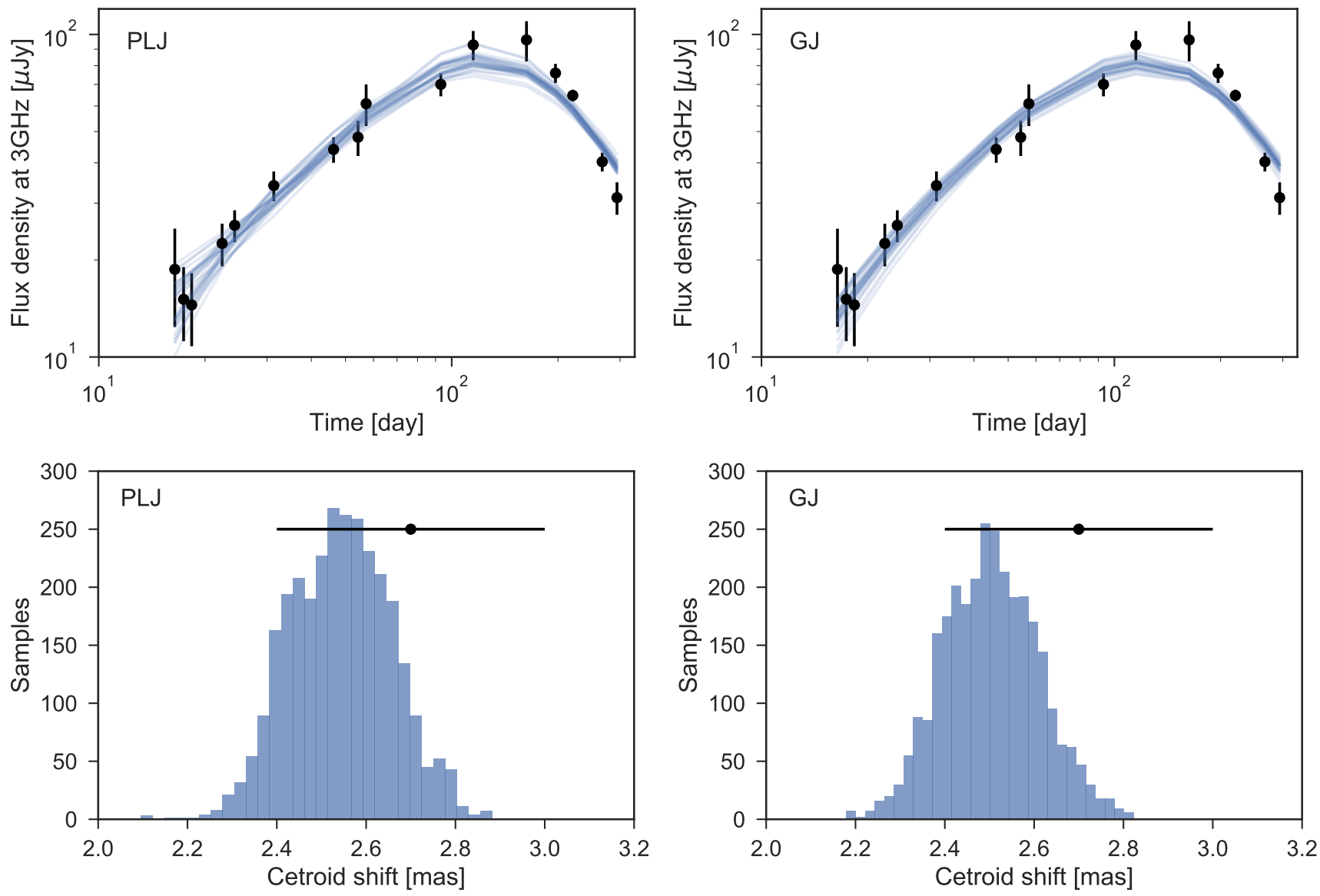

Figure 3: Afterglow light curve at $3 \mathrm{GHz}$ and centroid motion from day 75 to 230. Also shown are the light curves calculated with a PLJ (upper left) and a GJ model (upper right), where 50 sets of the model parameters are randomly chosen from the MCMC samples. Bottom panels show the histogram of the centroid motion with 3000 samples randomly chosen (lower left: a PLJ model and lower right: a GJ model). These are the results of the combined GW-VLBI-LC analysis. 


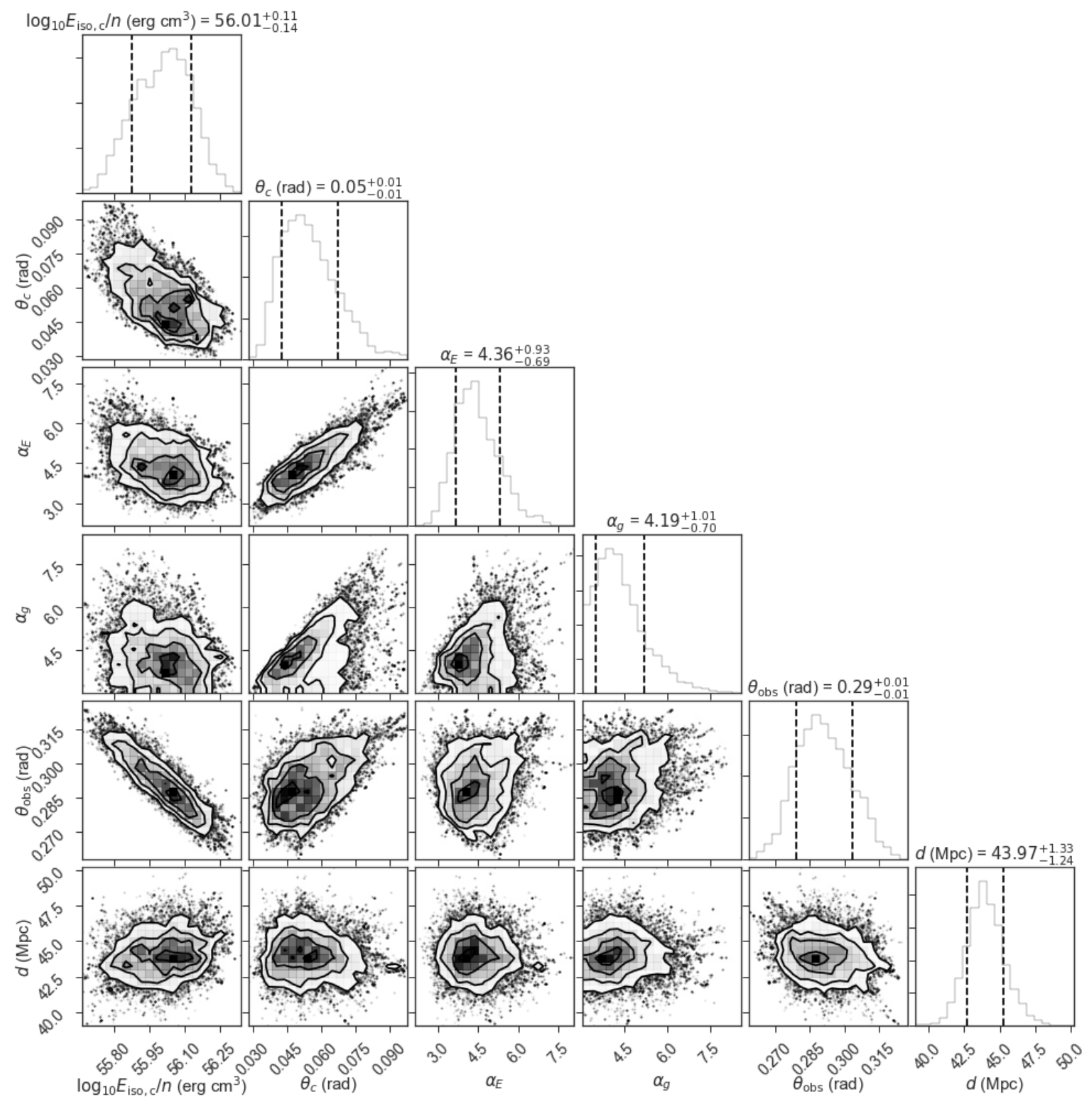

Figure 4: Corner plot for the combined GW-EM analysis with a Power-Law Jet model. The afterglow light curve at $3 \mathrm{GHz}$ and the centroid motion resolved by VLBI are used as the observed input data. Vertical lines depict $68 \%$ credible intervals. Here we use high spin PhenomNR posterior. 


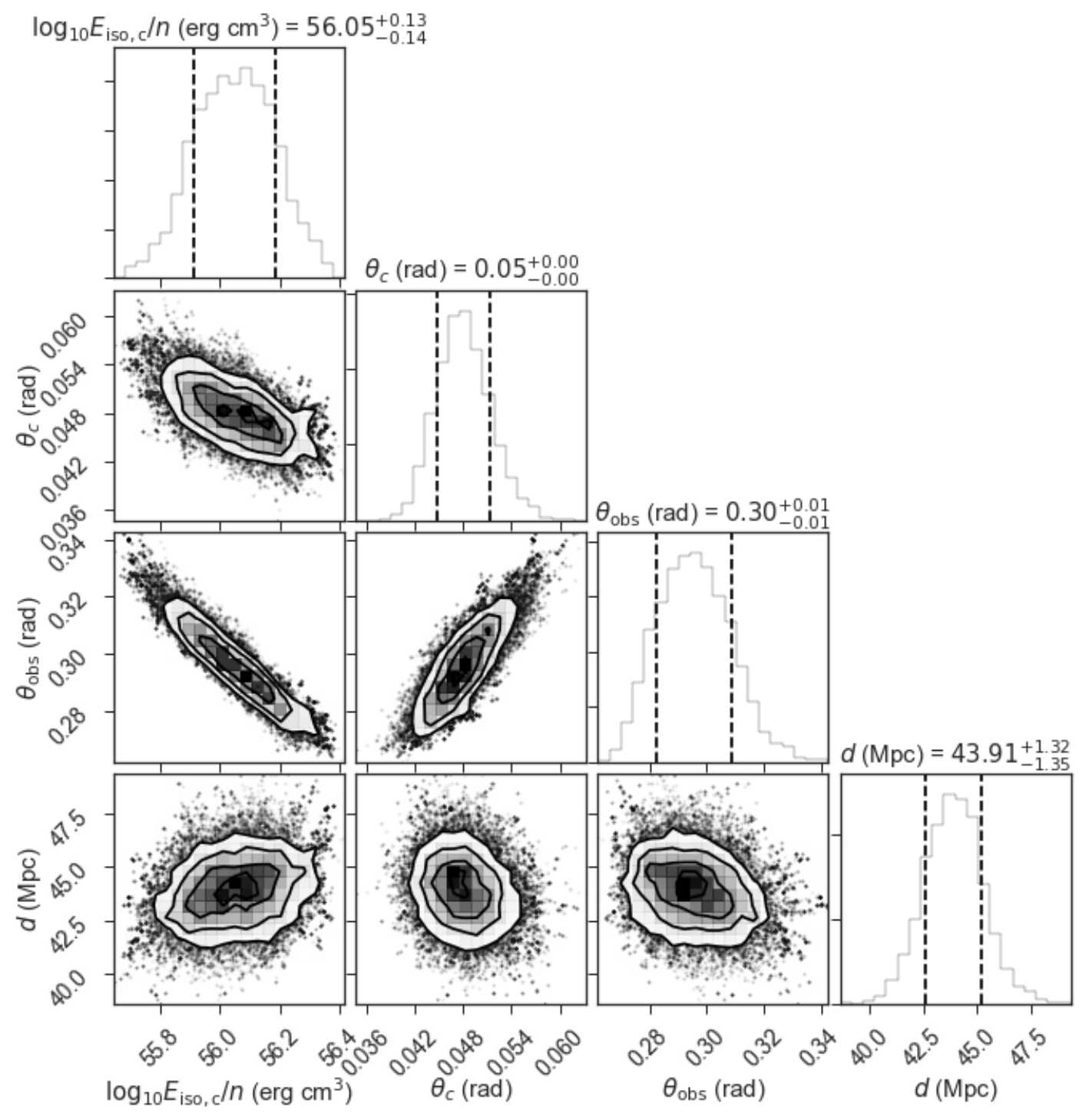

Figure 5: Same as Figure 4 but for a Gaussian Jet model. 


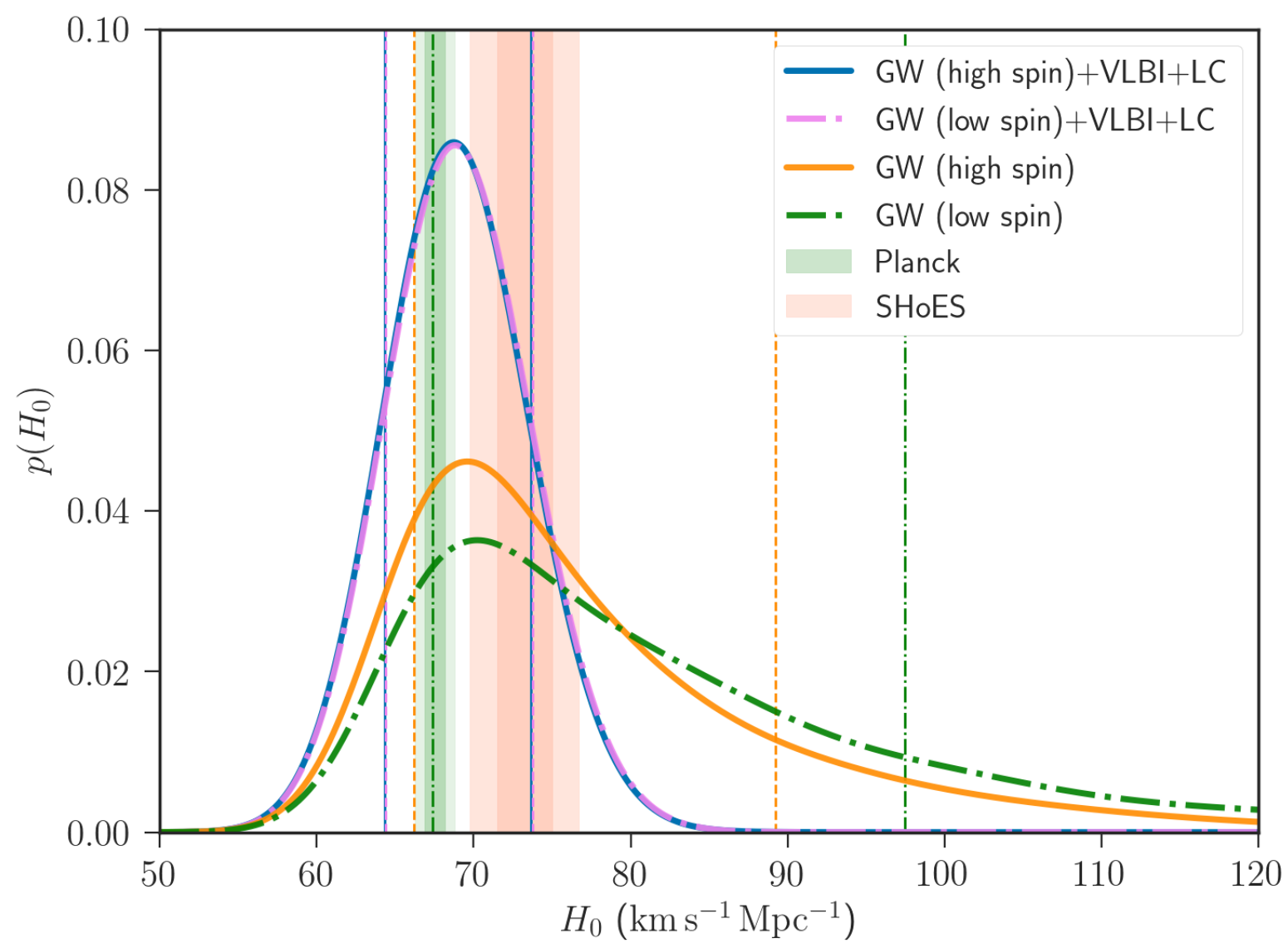

Figure 6: Comparison between the $H_{0}$ posteriors of the high and low spin priors. Here we use hydrodynamics simulation jet model $\left(0.25<\theta_{\mathrm{obs}}\left(\frac{d}{41 \mathrm{Mpc}}\right)<0.45 \mathrm{rad}\right)$. The vertical lines show symmetric $68 \%$ credible interval for each model. 


\section{References}

1. Hinshaw, G. et al. Nine-year Wilkinson Microwave Anisotropy Probe (WMAP) Observations: Cosmological Parameter Results. Astrophysical Journal Supplement 208, 19 (2013). 1212 . 5226.

2. Aubourg, É. et al. Cosmological implications of baryon acoustic oscillation measurements. Physical Review D 92, 123516 (2015). 1411.1074.

3. Planck Collaboration et al. Planck 2015 results. XIII. Cosmological parameters. Astronomy \& Astrophysic 594, A13 (2016). 1502.01589.

4. Riess, A. G. et al. A $2.4 \%$ Determination of the Local Value of the Hubble Constant. Astrophysical Journal 826, 56 (2016). 1604 . 01424.

5. Beaton, R. L. et al. The Carnegie-Chicago Hubble Program. I. An Independent Approach to the Extragalactic Distance Scale Using Only Population II Distance Indicators. Astrophysical Journal 832, 210 (2016). 1604 . 01788.

6. Gao, F. et al. The Megamaser Cosmology Project. VIII. A Geometric Distance to NGC 5765b. Astrophysical Journal 817, 128 (2016). 1511.08311.

7. Jang, I. S. \& Lee, M. G. The Tip of the Red Giant Branch Distances to Type Ia Supernova Host Galaxies. V. NGC 3021, NGC 3370, and NGC 1309 and the value of the Hubble Constant. ArXiv e-prints (2017). 1702.01118. 
8. Bonvin, V. et al. HOLiCOW - V. New COSMOGRAIL time delays of HE 0435-1223: $\mathrm{H}_{0}$ to 3.8 per cent precision from strong lensing in a flat $\Lambda$ CDM model. MNRAS 465, 4914-4930 (2017). 1607.01790.

9. Addison, G. E. et al. Elucidating $\Lambda$ CDM: Impact of Baryon Acoustic Oscillation Measurements on the Hubble Constant Discrepancy. Astrophysical Journal 853, 119 (2018). 1707.06547

10. DES Collaboration et al. Dark Energy Survey Year 1 Results: A Precise H0 Measurement from DES Y1, BAO, and D/H Data. ArXiv e-prints (2017). 1711.00403.

11. Riess, A. G. et al. Milky Way Cepheid Standards for Measuring Cosmic Distances and Application to Gaia DR2: Implications for the Hubble Constant. ArXiv e-prints (2018). 1804.10655 .

12. Feeney, S. M., Mortlock, D. J. \& Dalmasso, N. Clarifying the Hubble constant tension with a Bayesian hierarchical model of the local distance ladder. MNRAS 476, 3861-3882 (2018). 1707.00007

13. Schutz, B. F. Determining the Hubble constant from gravitational wave observations. Nature 323, 310 (1986).

14. Nissanke, S., Holz, D. E., Hughes, S. A., Dalal, N. \& Sievers, J. L. Exploring Short Gammaray Bursts as Gravitational-wave Standard Sirens. Astrophysical Journal 725, 496-514 (2010). 0904.1017. 
15. Abbott, B. P. et al. A gravitational-wave standard siren measurement of the Hubble constant. Nature 551, 85-88 (2017). 1710.05835.

16. Abbott, B. P. et al. GW170817: Observation of Gravitational Waves from a Binary Neutron Star Inspiral. Phys. Rev. Lett. 119, 161101 (2017). 1710.05832.

17. Abbott, B. P. et al. Multi-messenger Observations of a Binary Neutron Star Merger. Astrophysical Journal Letters 848, L12 (2017). 1710 . 05833.

18. Guidorzi, C. et al. Improved Constraints on $\mathrm{H}_{0}$ from a Combined Analysis of Gravitationalwave and Electromagnetic Emission from GW 170817. Astrophysical Journal Letters 851, L36 (2017). 1710.06426 .

19. Mooley, K. P. et al. Superluminal motion of a relativistic jet in the neutron star merger GW170817 (2018). 1806.09693.

20. Chen, H.-Y., Fishbach, M. \& Holz, D. E. Precision standard siren cosmology. ArXiv e-prints (2017). 1712.06531 .

21. Feeney, S. M. et al. Prospects for resolving the Hubble constant tension with standard sirens. ArXiv e-prints (2018). 1802.03404.

22. Hallinan, G. et al. A radio counterpart to a neutron star merger. Science 358, 1579-1583 (2017). 1710.05435.

23. Mooley, K. P. et al. A mildly relativistic wide-angle outflow in the neutron-star merger event GW170817. Nature 554, 207-210 (2018). 1711 . 11573. 
24. Ruan, J. J., Nynka, M., Haggard, D., Kalogera, V. \& Evans, P. Brightening X-Ray Emission from GW170817/GRB 170817A: Further Evidence for an Outflow. Astrophysical Journal Letters 853, L4 (2018). 1712 . 02809.

25. Alexander, K. D. et al. A Decline in the X-ray through Radio Emission from GW170817 Continues to Support an Off-Axis Structured Jet. ArXiv e-prints (2018). 1805.02870.

26. Margutti, R. et al. The Binary Neutron Star Event LIGO/Virgo GW170817 160 Days after Merger: Synchrotron Emission across the Electromagnetic Spectrum. Astrophysical Journal Letters 856, L18 (2018). 1801 . 03531.

27. Dobie, D. et al. A Turnover in the Radio Light Curve of GW170817. Astrophysical Journal Letters 858, L15 (2018). 1803.06853.

28. The LIGO Scientific Collaboration and the Virgo Collaboration et al. Properties of the binary neutron star merger GW170817. ArXiv e-prints (2018). 1805.11579 .

29. Abbott, B. P. et al. Prospects for observing and localizing gravitational-wave transients with Advanced LIGO, Advanced Virgo and KAGRA. Living Reviews in Relativity 21, 3 (2018). 1304.0670

30. Fong, W., Berger, E., Margutti, R. \& Zauderer, B. A. A Decade of Short-duration GammaRay Burst Broadband Afterglows: Energetics, Circumburst Densities, and Jet Opening Angles. Astrophysical Journal 815, 102 (2015). 1509.02922.

31. Nissanke, S. et al. Determining the Hubble constant from gravitational wave observations of merging compact binaries. ArXiv e-prints (2013). 1307.2638. 
32. Weinberg, D. H. et al. Observational probes of cosmic acceleration. Physics Reports 530, 87-255 (2013). 1201.2434 .

33. D'Avanzo, P. et al. The evolution of the X-ray afterglow emission of GW 170817/ GRB 170817A in XMM-Newton observations. Astronomy \& Astrophysic 613, L1 (2018). 1801. 06164

34. Troja, E. et al. The outflow structure of GW170817 from late-time broad-band observations. MNRAS 478, L18-L23 (2018).

35. Lamb, G. P. \& Kobayashi, S. GRB 170817A as a jet counterpart to gravitational wave triggerGW 170817. MNRAS 478, 733-740 (2018). 1710.05857.

36. Lyman, J. D. et al. The optical afterglow of the short gamma-ray burst associated with GW170817. ArXiv e-prints (2018). 1801.02669.

37. Gill, R. \& Granot, J. Afterglow Imaging and Polarization of Misaligned Structured GRB Jets and Cocoons: Breaking the Degeneracy in GRB 170817A. MNRAS (2018). 1803.05892.

38. Resmi, L. et al. Low frequency view of GW 170817/GRB 170817A with the Giant Meterwave Radio Telescope. ArXiv e-prints (2018). 1803.02768.

39. Nakar, E. \& Piran, T. Implications of the radio and X-ray emission that followed GW170817. MNRAS 478, 407-415 (2018). 1801.09712. 
40. Xie, X., Zrake, J. \& MacFadyen, A. Numerical simulations of the jet dynamics and synchrotron radiation of binary neutron star merger event GW170817/GRB170817A. ArXiv e-prints (2018). 1804.09345.

41. Gottlieb, O., Nakar, E. \& Piran, T. The cocoon emission - an electromagnetic counterpart to gravitational waves from neutron star mergers. MNRAS 473, 576-584 (2018). 1705 . 10797.

42. Lazzati, D. et al. Late time afterglow observations reveal a collimated relativistic jet in the ejecta of the binary neutron star merger GW170817. ArXiv e-prints (2017). 1712.03237.

43. Sari, R., Piran, T. \& Narayan, R. Spectra and Light Curves of Gamma-Ray Burst Afterglows. Astrophysical Journal Letters 497, L17-L20 (1998). a stro-ph/9712005.

44. Hotokezaka, K. \& Piran, T. Mass ejection from neutron star mergers: different components and expected radio signals. MNRAS 450, 1430-1440 (2015). 1501.01986.

45. Foreman-Mackey, D., Hogg, D. W., Lang, D. \& Goodman, J. emcee: The MCMC Hammer. Publications of the Astronomical Society of the Pacific 125, 306 (2013). 1202.3665.

46. Finstad, D., De, S., Brown, D. A., Berger, E. \& Biwer, C. M. Measuring the Viewing Angle of GW170817 with Electromagnetic and Gravitational Waves. Astrophysical Journal Letters 860, L2 (2018). 1804.04179 .

47. Cantiello, M. et al. A Precise Distance to the Host Galaxy of the Binary Neutron Star Merger GW170817 Using Surface Brightness Fluctuations. Astrophysical Journal Letters 854, L31 (2018). 1801.06080. 
48. Foreman-Mackey, D. corner.py: Scatterplot matrices in python. The Journal of Open Source Software 24 (2016). URL http://dx.doi.org/10. 5281 /zenodo.45906. 
Acknowledgements The authors are grateful to Duncan Brown, Christopher Hirata, Victoria Scowcroft, Peter Shawhan, David Spergel, Hiranya Peiris for useful discussions. We thank the LIGO Scientific Collaboration and Virgo Collaboration for public access to data products. K.H. is supported by Lyman Spitzer Jr. Fellowship at Department of Astrophysical Sciences, Princeton University. E.N. and O.G. are supported by the I-Core center of excellence of the CHE-ISF. SMN is grateful for support from NWO VIDI and TOP Grants of the Innovational Research Incentives Scheme (Vernieuwingsimpuls) financed by the Netherlands Organization for Scientific Research (NWO). The work of K.M. is supported by NASA through the Sagan Fellowship Program executed by the NASA Exoplanet Science Institute, under contract with the California Institute of Technology (Caltech)/Jet Propulsion Laboratory (JPL). GH acknowledges the support of NSF award AST-1654815. A.T.D. is the recipient of an Australian Research Council Future Fellowship (FT150100415).

Author Contributions $\quad$ K.H. carried out MCMC simulations with the synthetic models. E.N. and O.G. derived an analytic model and carried out hydrodynamic simulation to derive constraints on the viewing angle. K.H. and K.M. analyzed the posterior samples and calculated $H_{0}$. G.H, K.P.M., A.T.D. provided the input observational data. K.H., E.N., S.N., G.H. wrote the paper. All coauthors discussed the results and provided comments on the manuscript.

Competing Interests The authors declare no competing financial interests.

Correspondence Correspondence and requests for materials should be addressed to K.H. (email: kentah@astro.princeton.edu) and E.N. (email:udini@wise.tau.ac.il).

Data Availability $\mathrm{MCMC}$ samples are available from the corresponding author on request. 
Code Availability The codes used for generating the synthetic light curves are currently being readied for public release. Markov chain Monte Carlo Ensemble sampler: emcee. 